Special Issue of the 6th International Congress \& Exhibition (APMAS2016), Maslak, Istanbul, Turkey, June 1-3, 2016

\title{
Vibration Noise Harshness of a Light Truck Driveshaft, Analysis and Improvement with Six Sigma Approach
}

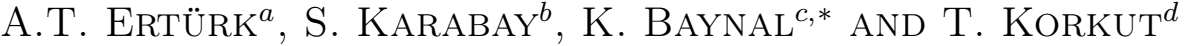 \\ ${ }^{a}$ Kocaeli University, Ford Otosan Ihsaniye Vocational School of Automotive, Kocaeli, Turkey \\ ${ }^{b}$ Kocaeli University, Department of Mechanical Engineering, Kocaeli, Turkey \\ ${ }^{c}$ Kocaeli University, Department of Industrial Engineering, Kocaeli, Turkey \\ ${ }^{d}$ Yazaki Corporation, Romania
}

\begin{abstract}
This paper deals with the application of six sigma methodology for optimization of a cardan shaft. The aim of this optimization is to reduce vibration of the drive shaft and consequently improve vibration noise harshness of the vehicle. The six sigma methodology has been applied to a light truck, which has received excessive vibration and noise complaints. The define-measure-analyze-improve-control approach has been followed to enhance the vibration noise harshness statistics. The result shows that all expected vibration noise harshness performance targets have been dramatically improved when compared to the initial values. As a conclusion, the case study on a light truck is a useful reference to improve vehicle performance for vibration noise harshness.
\end{abstract}

DOI: 10.12693/APhysPolA.131.477

PACS/topics: 06.30.Gv, 34.50.Ez, 43.58.+z, 46.40.-f, 05.10.-a

\section{Introduction}

The rotating shafts, typically referred as drive shafts, transmit torque from the engine to the driving wheels. There is a kinematic relationship due to the rotational position and velocity of the output shaft relative to the input shaft. Due to direction changes from applied moment to output moment, new moments are produced, as outlined in [1-3]. Further, physical kinematic relationship produces a second order, or two times per revolution, periodic angular speed fluctuations of the output shafts, despite the input driven at a constant rotational speed, through the non-constant velocity joint $[4,5]$. The difference between the input and output angular speeds has been defined as the cardan error for general rotodynamic applications $[1,6]$.

Increasing relative U-joint angle results in an increase in the amplitude of periodic fluctuations of velocity, based on the kinematic relationship. There has been significant research regarding the effects of single non-constant velocity joint systems. Porter $[7,8]$ has identified critical speed ranges, resulting in large amplitude oscillations for frequencies equal or for integer multiples of the system's natural frequencies, using both linear and nonlinear analysis. Porter $[8]$ has also predicted the generation of sub-harmonics as a possibility, but harmonics were deduced to be of lower amplitude, and thus less consequential. More recently, Chang [9] has identified the factor of the ratio of the input and output shaft stiffness influence on the torsional instability regions of Porter's single non-constant velocity joint system. Besides, Asokanthan et al. [10-12] have completed several analyses on torsional fluctuations due to non-constant velocity joints, including

*corresponding author; e-mail: kbaynal@kocaeli.edu.tr analysis of degrees of freedom of the system. These studies have addressed the torsional response of the same model, incorporating a single non-constant velocity joint system with fixed bearings. Mazzei et al. $[13,14]$ have identified parametric instabilities, including flutter due to the torsional and lateral response, produced by excitation of the non-constant velocity with applied driveshaft loading. Dimentberg $[15,16]$ has progressed the research into the soft support boundary conditions on the output shaft, including lateral, torsional and coupled response of the output shaft with support base excitation.

The cardan-type joint is extensively used in case of requirement that the input drive shaft and the output drive shaft are not aligned. It comprises three main parts including input drive shaft, output drive shaft and cross shaped piece. The angular speed of the input drive shaft is constant, whereas the output drive shaft oscillates. Thus, he ratio of the output velocity to the input velocity is not same for all angular positions [17]. Thus, misalignment is an essential source of vibration problems in vehicles. Cardan (Hooke's) joints provide flexibility to compensate or allow angular misalignments. The variation of joint moving angle is a response to force and moment inputs $[18,19]$. Angular misalignment gives rise to rotational speed variation between driveline segments. The output shaft speed fluctuates with the input shaft speed and misalignment angle [20].

Shudder symptoms indicate an engine-related case, which usually occurs during acceleration. The subsequent first low-frequency shaking action of the drivetrain system is closely related to the clonk response, known as shuffle. Shuffle appears at the first swing in rapid torque reversal. Repetitive clonk can happen at each cycle of shuffle under severe conditions [21].

The scope of this paper is the analysis of drumming noise generated by two-piece driveline, due to vibration during cruising and resolution/reduction of this noise 
with experimental study. This research has been done through determination of vibration transfer path using an accelerometer and a microphone (objective evaluation). After that determination/reduction of the vibration intensity by experimental analysis with six sigma method (subjective evaluation) was carried out. It has been analyzed using vibration noise harshness (VNH) testing equipment and reported with subjective evaluation techniques. After performing the detection stage of the VNH sources, reduction of vibration was investigated using the six sigma techniques. Non-constant velocity (universal joint), constant velocity joint and flex couplings have been used in driveshaft vehicle applications. The capability of a non-constant velocity is higher for transferring a significant torque value at relatively large misalignment degree $\left(5-10^{\circ}\right)$.

\section{Experimental procedure}

There are two factors which affect the problem. The first one is hanging bracket of the cardan shaft, and the other one is loading conditions. Cardan shaft humming problem is related with clonk, shuffle and shudder noises. Therefore, check points are defined as SGF (gearbox output shaft flange), UJ (universal joint) and pinion angles, indicated in Fig. 1.

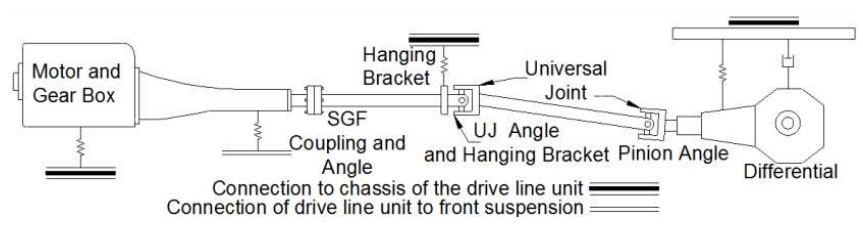

Fig. 1. Angles of the cardan shaft.

The key parameters related to the VNH source of the vehicle have been defined in Fig. 2. VNH measurements are handled in two stages, which are measurements on the outside and inside of the vehicle. In the study, vibration is defined as perceived movements of the rider at frequencies between 0.5 and $50 \mathrm{~Hz}$. To examine relationships between the the parameters using graphical analysis, the process capability analysis and data analysis were carried out at this stage. When the reasons are ordered according to their importance, they are categorized into hanger bracket of cardan shaft, axle pinion angle and seat angle of leaf springs categories. The analyzing procedure for the determination of the root cause of the problem was performed following the results outlined by the matrix indicated in Fig. 3. Analyses of these factors were carried out using the techniques defined in six sigma methodology and statistical problem-solving techniques. The purpose of this phase is to identify main causes of the trouble and to verify them.

Type of the microphones used in the measurements is the Brüel \& Kjaer 4189-A-021. During the test, the cabin windows were closed, and the passenger and the driver were provided with a quiet environment. The Brüel

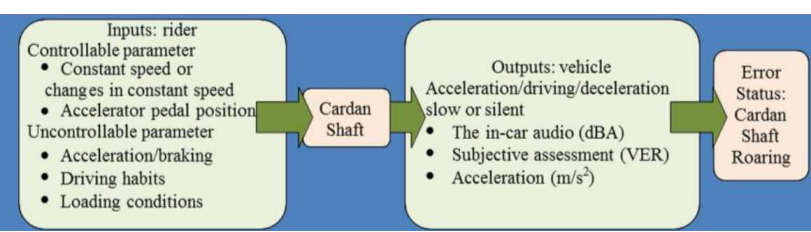

Fig. 2. The parameter diagram.

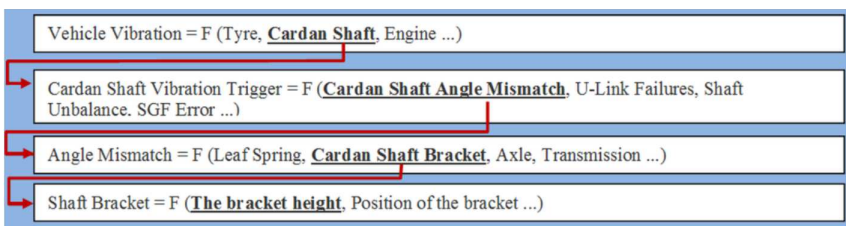

Fig. 3. The cause and effect analysis.

\& Kjaer microphone with type 2671 preamplifier was controlled and calibrated up to values of $93.8 \mathrm{~dB}, 1000 \mathrm{~Hz}$.

For collecting data from the driveline shaft, accelerometers were located on the shaft connecting blocks. Types and characteristics of the accelerometers were of 7254A-100 and 2258-100 type, with the sensitivity of $100 \mathrm{mV} / \mathrm{g}$ at $4 \mathrm{~mA}$. A tachometer was used to detect the vehicle speed.

After collecting the cabin sound level data and acceleration data, related to drive shaft connection places, it was transferred to an FFT analyzer for processing. The data was analyzed using Head Acoustics Artemis and SQ Lab-III computer software. In the tests, angles of six vehicle cardan shafts were measured, and sigma values for the currently available conditions were determined using Minitab. Objective data was received by slowly scanning in the range of $65-160 \mathrm{~km} / \mathrm{h}$, for the customary road conditions. Sound evaluation criteria of the cardan shaft are $20 \mathrm{~Hz}$ for the 1st harmonic and $400 \mathrm{~Hz}$ for the 2nd harmonic. Vibration evaluation criteria of the cardan shaft are $2 \mathrm{~Hz}$ for the 1st harmonic and $400 \mathrm{~Hz}$ for the 2 nd harmonic.

\section{Results and discussion}

The study of capability indices for SGF, UJ, and pinion angles was done for the following values, SGF angle $180 \pm$ $1^{\circ}$, height of the bracket $81.4 \mathrm{~mm}$. The six sigma values $Z$ and the number of defects per million opportunities (DPMO) have been computed. A convenient metric for summarizing the probability of a defect is the process capability as expressed by the $Z$ value. The calculated six sigma value of SGF angle of the unloaded vehicle was lower than the sigma value $(Z=-0.91)$ of the loaded vehicle. The calculated six sigma value of UJ angle $(Z=$ $-2.35)$ of the loaded vehicle was lower than the value $(Z=4.20)$ for the vehicles under unloaded condition. The calculated six sigma value of pinion angle $(Z=0.94)$ of the loaded vehicle was lower than the value $(Z=2.07)$ of the vehicle under unloaded condition. 
Data obtained from this study shows that the process capability $C_{\mathrm{pk}}$ value is related to SGF, UJ and pinion angles of unloaded and loaded cases of the vehicle. The $C_{\mathrm{pk}}$ shows considerable variation, and the $Z$ values are lower than those of the ideal situation and are dependent on it. In this case, it was decided to use the experimental design (DoE) to optimize the cardan shaft and the drive shaft angles. Here, the purpose of the DoE is to keep the desired values of the cardan shaft angles in the vehicle loaded and unloaded conditions. These angles have to be kept at optimum positioning values for the unloaded vehicle. On the contrary, when the vehicle is loaded, they have to remain within predetermined angle limits.

In the DoE, two factors were considered: (i) the height of cardan shaft with three levels (A height: $81.4 \mathrm{~mm}$; B height: $53.2 \mathrm{~mm}$; C height: $63.2 \mathrm{~mm}$ ), and (ii) loading conditions with two levels (I: vehicle not loaded with passengers and luggage, II: vehicle loaded, kerb weight+passengers+luggage). In these circumstances, a full factorial design was made to analyze the SGF, UJ and pinion angles.

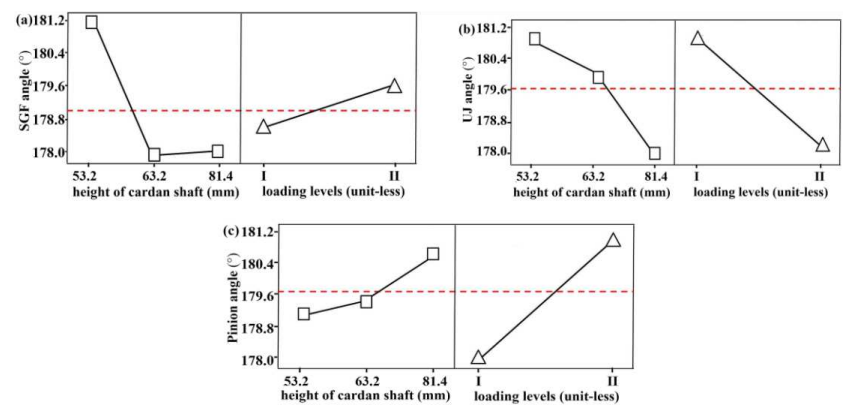

Fig. 4. Effects of hanger bracket and loading condition on the angles (a) SGF, (b) UJ, (c) pinion.

As a result of numerical indicators of $P=0.834>0.05$ for SGF angle, the interaction of the hanger bracket and loading condition were not effective on SGF angle. In technical terms, $P$-value is the probability of obtaining an effect. Therefore, the SGF parameter can be removed from DoE. Then the program was run again. The results were controlled to understand which factors more effective or ineffective. The value of $P$ for the hanger bearing was found to be $P=0$ for SGF angle. Thus, the DoE analysis revealed that the SGF angle was affected by the height of the cardan shaft bearing (see Fig. 4a).

In contrast, it has been determined that loading condition has no significant effect on the SGF angle (Fig. 4a). $P$ value for UJ angle is $0.859>0.05$. Thus, the interaction of hanger bracket and loading condition were not effective on the UJ angle. For this reason, this UJ angle parameter can be removed from DoE. The value of $P$ for the height of hanger bracket was found to be $P=0$. The DoE analysis has shown that the UJ angle was influenced by the cardan shaft hanger bed height. It was affected by loading condition for UJ angle (see Fig. 4b).

The $P$ value of combinational interaction of loading condition and hanger bracket was determined as $P=$
$0.323>0.05$ for pinion angle. This value means that there is no interactive relation between the loading condition and hanger bracket on the pinion angle. The $P$ value for hanger height came out of the DoE analysis as $P=0$ for pinion angle. It revealed that the pinion angle was affected by the hanger bracket of cardan shaft. Besides that, the loading status of the vehicle is also very effective on the pinion angle (see Fig. 4c).

Finally, the hanging bed height has been identified as the main factor in the DoE analysis. An empirical formulation has been constructed with statistical analysis using Minitab. Thus, it is possible to bring all the angles to desired values by changing of hanger bracket height. The ideal UJ angle for an acceptable level of VNH has to be 180 degrees. The graphs in Fig. 5 are constructed with evaluation of the multiple variations that have a specific indication for detecting the effects of all factors on the same graph. The effect of loading condition depending on the hanger bracket height on the SGF angle is shown in Fig. 5a.

The angle of SGF is affected by the hanger bracket height, but it is not affected by the changes in load. Loading effect over the UJ angle depending on the hanger bracket height is indicated in Fig. 5b. The angle of UJ has influenced both load condition and height of hanger bracket. Loading effect on the pinion angle depending on the height of the bracket is presented in Fig. 5c. It is influenced by changes of the load condition and of the height of hanger bracket. If the evaluation is performed with the help of data, it is derived that the optimum value for the angle UJ can be obtained with $63.2 \mathrm{~mm}$ height of the bracket. Moreover, SGF angle is not affected by loading conditions of the vehicle.

Hanger bracket height $(\mathrm{mm}) \triangle: 81.4, \bigcirc: 63.2, \square: 53.2$

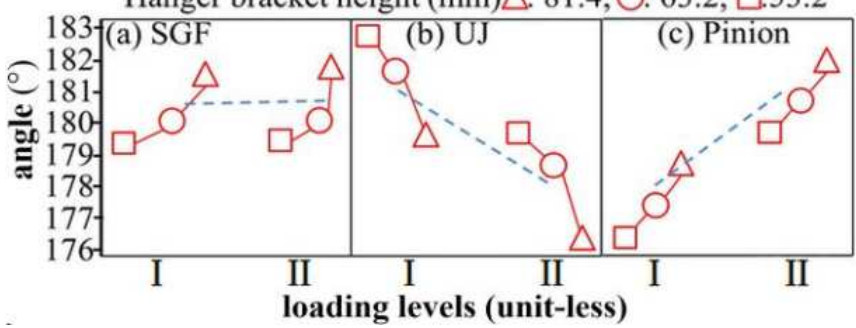

Fig. 5. Multiple variations assessment, effect of hanger bracket and loading (a) SGF, (b) UJ and (c) pinion angle.

Objective evaluation was performed with an accelerometer, microphone, data analyzer and tachometer, and the following results were obtained. In the case where the engine reaches its regime, the test was performed with very smooth acceleration in the range of $115-120 \mathrm{~km} / \mathrm{h}$ at the highest gear and was repeated six times. The accelerometer mounted onto the gearbox (Fig. 6a), the center ball bearing (Fig. 6b) and the pinion (Figure 6c) is indicated in Fig. 6. In Fig. 7 the darker color points out the decreasing level of the noises in spectra. Noise chart 
was created by data analyzer for verification. Minimized noise level of the objective measurements was achieved with $63.2 \mathrm{~mm}$ hanger bracket height, which was determined by using the DoE improvement analysis.
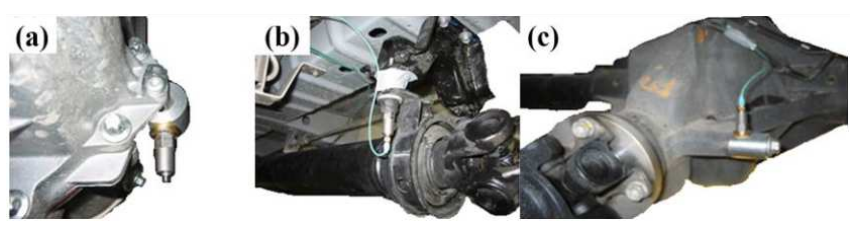

Fig. 6. Mounting positions of accelerometers on (a) the gearbox, (b) center ball bearing and (c) the pinion.

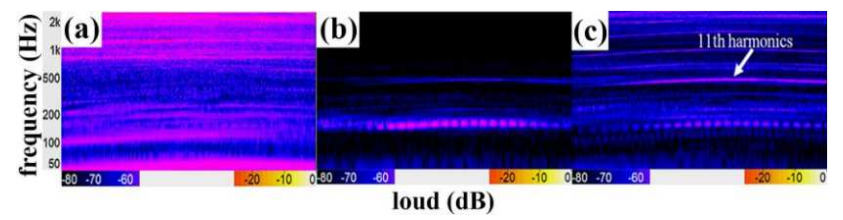

Fig. 7. Noise spectrum recorded from (a) the gearbox,

(b) center ball bearing and (c) the pinion.

Figure 8 shows the improvement of $5 \mathrm{~dB}$ of the cardan shaft hum at $150 \mathrm{~Hz}$ critical frequencies. Under this condition, a traveler does not distinguish noises (humming) generated by a cardan shaft, because an upper volume limit arising from cardan shaft is defined as $65 \mathrm{~dB}$ for a commercial vehicle. Also, the new bracket performance is valid not only for $150 \mathrm{~Hz}$. It provides decreasing effects at all frequencies. This result is also consistent with results obtained from subjective reviews.

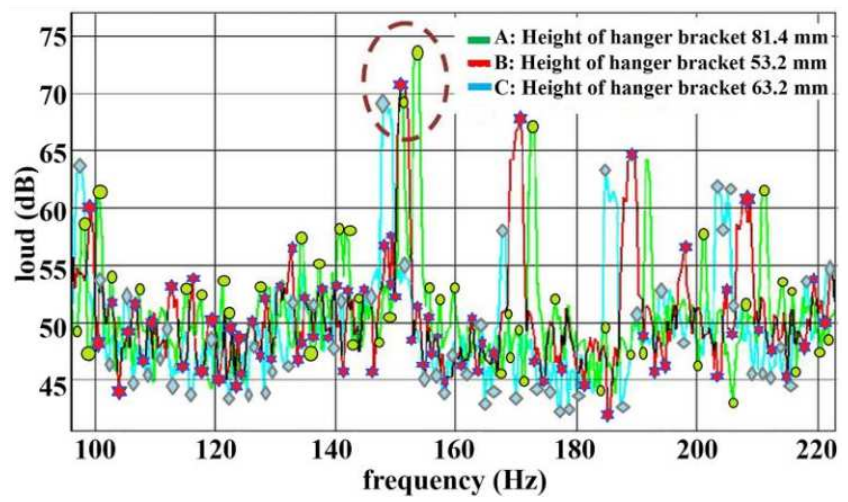

Fig. 8. The fast Fourier transform (FFT) graph.

\section{Conclusions}

Many previous investigations of $\mathrm{VNH}$ have focused on the theoretical analysis of driveline dynamics. The novelty of this paper is to comprehensively investigate and evaluate the relationships between using/driving habits and VNH problems. Effects of loading conditions and acceleration/deceleration on NHV problems have been identified using experimental techniques and a real case study analysis. Cardan shaft vibration, affecting the entire vehicle sound quality, is one of the most important sources of VNH. In the subjective assessment, there was no noise problem, caused by the cardan shaft with C-type hanger bracket. Noise level with C-type hanger bracket was determined as optimum condition. Hanger bracket changing from $81.4 \mathrm{~mm}$ (A-type) to $63.2 \mathrm{~mm}$ (C-type) has provided the significant improvement in noise problem, which was detected with DoE and subjective assessment. Objective data confirm this improvement.

\section{References}

[1] I. Porat, Mech. Mach. Theory 15, 245 (1980).

[2] J.E. Baker, Mech. Mach. Theory 37, 1127 (2002).

[3] I. Holsteins, J. Anthonis, H. Ramon, Mech. Syst. Signal Pr. 19, 105 (2005).

[4] E.R. Wagner, C.E. Cooney, in: Universal Joint and Drive Shaft Design Manual, Eds. E.R. Wagner, SAE International, Warrendale 1979, p. 39.

[5] A.H. Berker, in: Universal Joint and Drive Shaft Design Manual, Eds. E.R. Wagner, SAE International, Warrendale 1979, p. 407.

[6] H.A. DeSmidt, K.W. Wang, E.C. Smith, J. App. Mech. 69, 261 (2002).

[7] B. Porter, J. Mech. Eng. Sci. 3, 324 (1961).

[8] B. Porter, Proc. R. Soc. A 277, 92 (1964).

[9] S.I. Chang, J. Sound Vib. 229, 993 (2000).

[10] S.F. Asokanthan, P.A. Meehan, J. Sound Vib. 233, 297 (2000).

[11] S.F. Asokanthan, M.C. Hwang, J. Vib. Acoust. 118, 368 (1996).

[12] S.F. Asokanthan, X.H. Wang, J. Sound Vib. 194, 83 (1996).

[13] A.J. Mazzei Jr., R.A. Scott, J. Sound Vib. 244, 555 (2001).

[14] A.J. Mazzei Jr., A. Argento, R.A. Scott, J. Sound Vib. 222, 19 (1999).

[15] M.F. Dimentberg, Int. J. Nonlinear Mech. 40, 1263 (2005).

[16] M.F. Dimentberg, Int. J. Nonlinear Mech. 40, 711 (2005).

[17] H.W. Chen, W.X. Ji, Q.J. Zhang, Y. Cao, S.Y. Fan, J. Mech. Sci. Technol. 26, 335 (2012).

[18] F.L. Barber, M.Sc. Thesis, Massachusetts Institute of Technology, Cambridge 1988.

[19] T. Iwatsubo, M. Saigo, J. Sound Vib. 95, 9 (1984).

[20] H.A. DeSmidt, Ph.D. Thesis, Pennsylvania State University, Pennsylvania 2005.

[21] A. Farshidianfar, M. Ebrahimi, H. Rahnejat, M.T. Menday, M. Moavenian, J. Mech. Eng. Sci. 216, 249 (2002). 